\title{
CHALLENGES IN MANAGING SARS-COV-2 POSITIVE CHILD WITH JUVENILE ONSET RECURRENT RESPIRATORY PAPILLOMATOSIS: A CASE REPORT WITH REVIEW OF LITERATURE
}

\author{
Heempali Das Dutta ${ }^{1}$ Bibhu Pradhan ${ }^{2 *}$ \\ ${ }^{1}$ Dr. Dutta is an Assistant Professor; and ${ }^{2}$ Dr. Pradhan is Professor and Head, \\ Department of ENT\& HNS, Institute of Medicine, Tribhuvan University Teaching Hospital, \\ Maharajgunj Medical Campus, Kathmandu, Nepal \\ *Corresponding Author: bibhuduga@yahoo.com
}

\begin{abstract}
Juvenile-Onset Recurrent Respiratory Papillomatosis (JORRP) is a viral infection caused by Human papilloma virus 6, 11 which presents as hoarseness, shortness of breath and in severe form may present with acute airway obstruction because of papillomatous growth obstructing the larynx. Urgent surgical intervention may be needed to secure the airway. In the present situation of Coronavirus disease-2019 (COVID-19) pandemic, the case of JORRP with COVID-19 positive state is a challenging situation because any laryngeal or airway surgery is associated with high amount of aerosol generation. Air borne transmission is one of the major routes of transmission for SARS-Cov-2 virus which may result in spread of COVID-19 among to the health care provider.

In this situation, it is better to avoid delay laryngeal surgery if the patient is not in acute airway obstruction requiring urgent surgical intervention. Otherwise, if the urgent surgery has to be done, then it should be done properly by following institutional guidelines to avoid the spread of the disease and to be done with all precautionary biosafety measures like use of personal protective equipment (PPE), N95 mask and proper donning and doffing.
\end{abstract}

Keywords: JORRP, airway obstruction, COVID-19 


\section{INTRODUCTION}

Juvenile-Onset Recurrent Respiratory Papillomatosis (JORRP) is a potentially life threatening disease characterized by the development of papillomata anywhere in respiratory tract from the nasal vestibules to the terminal bronchi. Predominant sites are vocal cords, tonsillar pillar, uvula where there is change of epithelium. The virus can be detected in the normal mucosa adjacent to lesions which provides a reservoir for regeneration of new papillomata. JORRP was first described by Morrell and Mackenzie in 1880 (Rania et al 2018). The causative organism is Human papilloma virus types 6 and 11 (Mount et al 1982). The estimated incidence is 2 per 100,000 in adults and 4 per 100,000 in children (William, 2003). JORRP commonly present between two to four years of age with hoarseness, which may progress to stridor, increase work of breathing and eventually, complete airway obstruction (Derkay et al 2006; Stamataki et al 2007). There is no known cure for JORRP. The child may require multiple surgical excisions may be up to 30 times. There are various types of treatment available like surgical excision, antiviral therapy, immunotherapy, human papilloma virus vaccine etc but surgery remains the mainstay of treatment. The main aim of surgery is complete removal of the papillomas and preservation of the normal structures. During surgical excision, most of the papillomatous mass can be excised under direct vision with the help of rigid endoscope or microscope. The preferred methods are resection using microdebrider or $\mathrm{CO}_{2}$, Nd: YAG or pulse-dye Laser (Kaalan Johnson et al 2009). At the present, situation of COVID-19 pandemic, the laryngeal procedure is not without risk as it is associated with high amount of aerosol generation. Clarie et al in 2020 suggested that all pediatric patients undergoing urgent otolaryngology procedures should be treated as suspected COVID-19 cases until proven otherwise due to the higher proportion of asymptomatic children carrying COVID-19. Management of child with JORRP in acute airway obstruction with COVID-19 positive state is one of the challenging situations.

\section{CASE REPORT}

A 6 years old girl from Kapilvastu District Nepal visited Out Patient Department (OPD) of ENT and HNS, TUTH, Kathmandu on 3 Bhadra, 2075 BS. and was diagnosed as a case of JORRP. She underwent 
endoscopic excision JORRP thrice at TUTH. The last excision was done on 8 Mangsir, 2076 BS. and preoperative finding was papillomatous growth arising from laryngeal surface of epiglottis, bilateral aryepiglottic folds, arytenoids, true and false vocal cords, anterior commissure and posterior commissure.

Five months after the surgery, child presented in ENT OPD on 30 Baishakha, 2077 BS. after screening in the fever clinic, she gave the history of worsening of hoarseness for last 1 month and noisy breathing for last 1 week. There was no history of fever, cough and shortness of breath. There was no history of travel abroad. There was no family history of above symptoms or travel history. On examination, patient was in inspiratory stridor with vitals within normal including Spo2 96\% at room air.

Laryngeal examination with nasopharyngeal laryngoscopy (NPL) was avoided as the child was a known case of JORRP and moreover NPL is considered as aerosol generating procedure. An endoscopic excision of JORRP under general anesthesia as routine planned surgery following PCR negative report for SARS- CoV-2 is available, as the patient is from a known COVID-hot spot in present context and the endoscopic excision is an aerosol generating procedure requiring adequate biosafety protection. She was admitted in the ward, on waiting for the surgery.

On the next day, Real Time PCR test report came to be positive for SARS-Cov-2 (COVID-19). So the child was shifted to the isolation ward. After a course of antibiotics and steroid, the stridor improved and child was stable without any respiratory distress features of COVID-19.

Therefore, emergency surgery was postponed for two weeks and child was kept in quarantine. The further plan of treatment is to do endoscopic excision under general anaesthesia once her SARS-Cov-2 PCR becomes negative after 14 days.

\section{DISCUSSION}

JORRP is an emergency condition requiring urgent surgical excision because of impending threat of acute airway obstruction. In the present situation of COVID-19 pandemic, managing the JORRP in COVID-19 positive patient is very challenging. ENT procedures like oropharyngeal 
examination, laryngoscopy, NPL and surgical excision is considered as an aerosol generating procedures.

Within an aerosol, viral droplet nuclei can travel long distances and remain in the air for long periods of time as postulated by Jones et al 2015. Aerosols are not as effectively filtered by surgical masks, and can be breathed directly into the lungs. Evidence indicates that betacoronaviradae such as the 2003 SARS Coronavirus (SARS-CoV-1) are viable in aerosols (Jones et al 2015). COVID-19 RNA was detected in air samples in hospital rooms of patients with COVID-19 as Santarpia et al (2020) mentioned in their study. A widely cited experimental study by Van Doremalen et al in 2020 indicates that COVID-19 can remain viable in aerosols for hours.

In this case, the child did not have any symptoms of $S A R S-C o v-2$ infection. She had shortness of breath because of papillomatous growth which was obstructing her airway. Current data of Lu X et al study in 2020, suggests that $15 \%$ paediatric COVID-19 positive cases are asymptomatic and $25 \%$ present with features of an upper respiratory tract infection. The viral load of asymptomatic paediatric patients is considered to be very high in the study done by Kam et al in 2020. Considering above knowledge and her demographic information showing her residence origin from one of the COVID-19 hot spot area, RT PCR test for SARS-Cov-2 was requested. The patient was treated symptomatically because she was not in acute airway obstruction, based on standard protocol.

Recent publications have shown that high number of health care workers including otolaryngologists, in particular, have been infected during the course of their recent clinical work ( $Z$ Patel et al, Lu D et al 2019, Wang et al 2019). Therefore, we postponed the surgery for 14 days because viral shedding and transmission may occurs during the period in which the median incubation time of SARS-CoV-2 is five days as shown in the study done by Laner et al 2020 and Bai et al 2020. Laner et al 2020, study found that among COVID-19 positive patients who eventually develop symptoms, $99 \%$ have been out of symptoms by $14^{\text {th }}$ day.

Moreover, surgical excision of JORRP requires direct visualization and manipulation and instrumentation of larynx and lower airway with microdebrider, coblation and other cold instruments and thus may be 
associated with aerosol generation. In addition to this, surgeries performed with general anesthesia involve multiple, routine aerosol-generating procedures such as bag-valve mask ventilation and intubation (Tran et al 2012). Surgeries involving the upper aerodigestive tract are at high-riskfor SARS-CoV-2 viral aerosol generation in the operating room including risk to health care professionals involved in the process as postulated by Tran et al 2012 and Judson et al 2019.

In this case, the postponement of the surgery was possible as the child was not in acute respiratory distress. However, in situation where urgent lifesaving surgeries required like micro-laryngoscopic excision of JORRP, adequate biosafety and protective measures have to be in place. Proper planning and institutional biosafety guideline has to be followed. Paul Mick et al in 2020 suggested that during the COVID-19 pandemic, proper use of PPE by the health care workers is critical in reducing transmission of the infection in health care settings, particularly when aerosol-generating medical procedures are being performed. So it is recommended to minimize aerosol generating procedures and limit the length of these procedures whenever possible. For example, anesthesia may consider avoiding bagmask ventilation, employing rapid sequence intubation techniques, and applying intratracheal or intravenous lidocaine to avoid postoperative coughing (Cui et al 2019, Yang et al 2020). Hus et al in 2020 suggested that team involved in surgery also requires to be trained in appropriate use of respirators and enhanced PPE donning and doffing procedures.

\section{CONCLUSION}

To conclude, the cases of JORRP presented with acute respiratory distress should be evaluated for COVID-19 status and should be managed properly. In this situation, our aim should be to treat the acute airway obstruction and at the same time to take proper measures to avoid cross infection to health care professionals. 


\section{REFERENCES}

Bai, Y., Yao, L. \& Wei, T., (2020). Presumed asymptomatic carrier transmission of Covid-19. Journal American Medical Association, 323(14):1406-1407.

Claire, F., Colin, B., Ben, H., Lesley, C., Chris, J., Robert, N., et al. (2020). Practical insights for paediatric otolaryngology surgical cases and performing microlaryngobronchoscopy during Covid 19 pandemic. International Journal of Paediatric otolaryngology, 134:110030.

Cui, Y., Wang, Y., Cao, R., Liu, K., Huang, Q.H. \& Liu, B. (2019). On-table extubation in neonates undergoing anoplasty: An experience of anesthetic management on the concept of fast-tracking anesthesia: A pilot study. Medicine, 98(2):14098.

Derkay, C.S. \& Darrow, D.H. (2006). Recurrent respiratory papillomatosis. Annal of Otol Rhinol Laryngol, 115: 1-11.

Huh, S., (2020). How to train the health personnel for protecting themselves from novel coronavirus (COVID-19) infection during their patient or suspected case care. J EducEval Health Profess, 17:10.

Jones, R.M. \& Brosseau, L.M., (2015). Aerosol transmission of infectious disease. Journal of Occup Environ Med, 57(5):501-8.

Judson, S.D. \& Munster, V.J., (2019). Nosocomial transmission of emerging viruses via Aerosol-generating medical procedures. Viruses, 11(10).

Kaalan J. \& Craig D. (2009). Palliative aspects of recurrent respiratory papillomatosis. Otolaryngol Clin N Am, 42:57-70.

Kam, K.Q., Yung, C.F., Cui, L., Pin, R.L.T., Mak, T.M., Maiwald, M., et al. (2020). A well infant with coronavirus disease 2019 (COVID-19) with high viral load. Clin. Infect. Dis.: An Official Publication of the Infectious Diseases Society of America, 361:1701.

Lauer, S.A., Grant, K.H., Bi, Q., (2020). The incubation period of Coronavirus Disease 2019 (COVID-19) from publicly reported confirmed cases: estimation and application. Ann Internal Med. doi: 10.7326/M20-0504.

Lu, D., Wang, H., Yu, R., Yang, H. \& Zhao, Y. (2020). Integrated infection control strategy to minimize nosocomial infection of coronavirus 
disease 2019 among ENT health care workers. Journal of Hospital Infection, 104(4):454-455.

Lu, X., Zhang, L., Du, H., Zhang, J., Li, Y.Y., Qu, J., et al. (2020). Team SARS-CoV-2 infection in children. The New England Journal of Medicine, 382(17):1663-1665

Mounts, P., Shah, K.V. \& Kashima, H., (1982). Viral etiology of juvenile and adult-onset squamous papilloma of the larynx. Proceeding of National Academy of Science, 79: 5425-9.

Paul, M. \& Russell, M. (2020). Aerosol-generating otolaryngology procedures and the need for enhanced PPE during the COVID-19 pandemic: a literature review. Journal of Otolaryngology - Head \& Neck Surgery, 49(29).

Rania, M. \& Michael, K., (2018). Juvenile-onset recurrent respiratory papillomatosis. In: John CW \& Raymond WC (eds.). Scott Brown's otorhinolaryngology Head and Neck Surgery. $8^{\text {th }}$ ed. London: CRC Press, pp. 367-376.

Santarpia, J.L., Rivera, D.N., Herrera, V., Morwitzer, M.J., Creager, H., Santarpia, G.W., et al. (2020). Transmission potential of SARSCoV-2 in viral shedding observed at the University of Nebraska Medical Center. MedRxiv. 20039446.

Stamataki, S., Nikolopoulos, T.P. \& Korres, S. (2007). Juvenile recurrent respiratory papillomatosis: still a mystery disease with difficult management. Head Neck, 29(2):155-162.

Tran, K., Cimon, K., Sever, M., Pessoa-Silv, C.L. \& Conly, J., (2012). Aerosol generating procedures and risk of transmission of acute respiratory infections to healthcare workers: a systematic review, 7(4):e35797.

VanDoremalen, N., Bushmake, T., Morris, D.H., Holbrook, M.G. \& Gamble, A. (2020). Aerosol and Surface Stability of SARSCoV-2 as Compared with SARS-CoV-1. New England Journal of Medicine, 382(16):1564-1567. 
Wang, D., Hu, B., Hu, C., Zhu, F., Liu, X., Zhang, J., et al. (2020). Clinical characteristics of 138 hospitalized patients with 2019 novel coronavirus-infected pneumonia in Wuhan, China. J. Am. Med. Assoc. 323:1061

William, C.R., Snehal, S.R., Katherine, I.S., Craig, S., Avron, M. \& Elizabeth, R.U. (2003). National Registry for Juvenile-Onset Recurrent Respiratory Papillomatosis. Archives of Otolaryngology Head Neck Surgery. 129: 976-82.

Yang, S.S., Wang, N.N. \& Postonogova, T., (2020). Intravenous lidocaine to prevent Postoperative airway complications in adults: a systematic review and meta-analysis. British Journal of Anaesthesia. 124(3):314-323.

Z Patel, J., Fernandez-Miranda, P., Hwang, J.V., Nayak, R., Dodd, H. \& Sajjadi, R. (2019). Precautions for endoscopic transnasal skull base surgery during the covid-19 pandemic, Neurosurgery, in-press. 